\title{
Spacetime: A Distinct Medium
}

\author{
AC Tahan ${ }^{1}$ \\ ${ }^{1}$ ETHERMED $_{\mathrm{Tм}}$, Cambridge, MA, USA \\ Correspondence: AC Tahan, ETHERMED, PO Box 391987, Cambridge, MA 02139, USA. E-mail: \\ actahan@ethermed.com
}

\author{
Received: September 30, 2014 Accepted: October 19, 2014 Online Published: November 25, 2014 \\ doi:10.5539/esr.v4n1p40 \\ URL: http://dx.doi.org/10.5539/esr.v4n1p40
}

\begin{abstract}
String experimentation has allowed for the observation in the laboratory of a D-brane with an open string for visible light (Tahan 2011). Events in experiments when the best defined, largest D-brane appeared suggested the subsistence of a separate medium that was concluded to be spacetime since no other extra-dimensional environment in which light travels could be imagined. Specifically, a high gravitational area in the tube of the set-up rotating and consequently dragging the background in which the light existed causing deformation of the D-brane with the open string (Tahan 2011) triggered the idea of an additional setting. The observation was in addition to an initial pushing influence that was later concluded to have affected the medium thereby exposing the D-brane and open string by seemingly stopping the laser light in place, rather than having influenced the light directly. This manuscript discusses environmental conditions in experiments that substantiated existence of a distinct medium. Understanding spacetime as a particular extra-dimensional environment does not require the altering of Special or General Relativity since related laws do not change. This paper is not reintroducing the classical, absolute aether but is expressing the existence of a general relativity aether or spacetime; the same explanations for mass influencing spacetime particularly for gravity do not change. This work simply proposes spacetime to be a tangible environment to which properties can be transferred.
\end{abstract}

Keywords: spacetime, general relativity aether, graviton, graviphoton

\section{Introduction}

Strings have been recorded (Tahan 2011) due to a tool that allows for studies outside the Standard Model. The instrument allowing for string experimentation (Tahan 2014) permits access to the theoretically predicted fifth dimension (Kaluza 1921, Klein 1926) resulting in the appearance of gravitons and superparticles in the dimensions of the Standard Model. Specifically, experimentation has supported that a separation between the visible sector and an underlying region of superparticles including the graviton exists and the area of superparticles and gravitons can be accessed through symmetry breaking involving the strings of the Hydrogen brane (Tahan 2014), permitting additionally the observation of hidden structures as D-branes--indicative of an extra dimension.

The most visible, largest D-brane with an open string was recorded when barometric pressure and relative humidity were about $99.7 \mathrm{kPa}$ and $98-100 \%$ (Boston, MA, CW1378) (Tahan 2011). Barometric pressure being low while relative humidity was high seemed to be consistent, ideal requirements to observe D-branes and open strings for the laser light when in the set-up (Tahan 2014). The low barometric pressure has been explained to be advantageous since less pressure against the tube due to the atmosphere permitted influences more easily to emerge from the vessel and affect surroundings (Tahan 2011).

Pushing and pulling influences, observed on matter in experiments, were understood emerging due to the string experimentation process. A pushing influence typically preceded a pulling affect that was associated with the emergence of gravitons. Pushing and pulling were only to describe movements between objects, i.e. pushing meaning movement away from the graphite tube and pulling being a progression to the vessel. The observations were concluded due to particles resulting from the symmetry breaking process (Tahan 2012) interacting with the tube and other objects as a pipette if incorporated in the set-up, e.g. gravitons imparting mass-energy. The imparting would cause the matter to affect spacetime thereby resulting in observed movement: progression toward each other due to the tube bending spacetime because of transferred graviton mass-energy. Or bodies seemed to move apart due to a relaxed influence on spacetime resulting from an opposite affect from the tube 
caused by a particle that was influential usually before the graviton, concluded with trials to have been likely the graviphoton. Objects were thought also to radiate imparted graviphoton or graviton energy so that a body that may not have interacted with the original graviton or graviphoton could have had radiated energy interact with it. The object would be affected similarly to having been in direct contact with the superparticle or graviton that had initially imparted influence to the radiating body.

The notion of spacetime emerged when Special Relativity was presented in the context of a four coordinate system (Minkowski 1908, Minkowski 1909). Consequently, distinguishing space separately from time became reserved for non-relativity discussions. The spacetime of this manuscript should not be thought to encourage the notion of the classical, mechanical aether. The environmental conditions of the experiment allowing for the observation of the clearest and largest D-brane with an open string supported that an extra-dimensional, non-absolute medium exists to which properties related to mass--specifically in consideration of $\mathrm{E} / \mathrm{c}^{2}=\mathrm{m}$ (Einstein 1905)--can be transferred. Therefore, the spacetime of this work is not dissimilar to the description of the aether of general relativity (Einstein 1920, Einstein 1924) that largely has been forgotten. Acceptance of a specific spacetime medium or relativistic aether among physicists has been unpopular (Laughlin 2005), particularly due to past work that discredited the existence of the classical aether with a state of motion (Michelson \& Morley 1887, Shankland, R.S. 1964). This work is a first presentation due to experimentation for the existence of a specific spacetime medium or general relativity aether, a tangible environment.

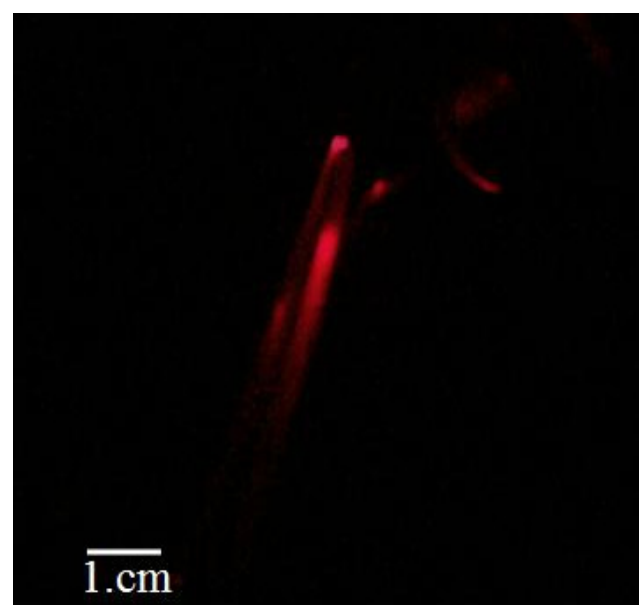

a

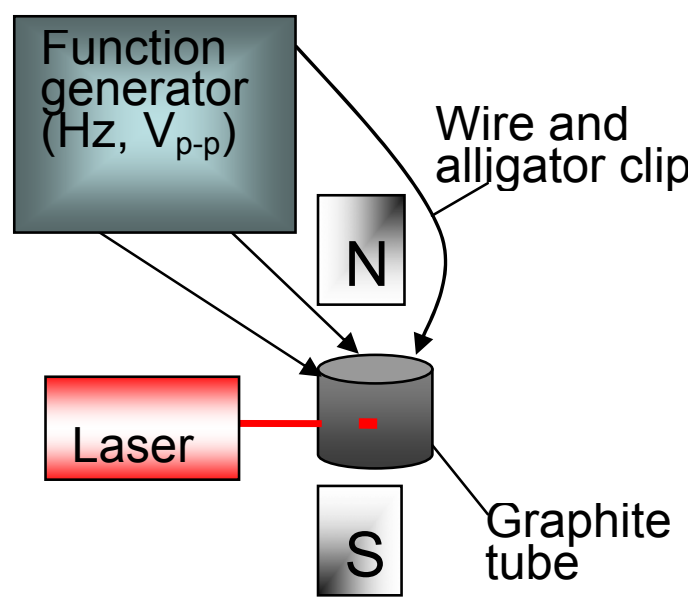

b

Figure 1. D-brane with an open string and the instrument with the laser

a. The appearance of the D-brane with an open string for the laser light that was incorporated with the string experimentation instrument (Tahan 2011) is shown. To improve opportunities to see D-branes with open strings similar to the Figure 1a D-brane new graphite tubes should be used because more graphite atoms will leak to the acid, where gravitons with superparticles emerge and will encounter the atoms. The resulting high gravitational area, black hole, would be a concentrated graviton and superparticle energy source allowing for greater pushing and pulling influences. Without the novel tubes, D-branes can still be seen if having low barometric pressure and high relative humidity but not as vividly or large as the Figure 1a D-brane and open string.

b. Laser light (Quartet Standard Laser Pointer), $\approx .017 \mathrm{~m}$ from the middle and about $.01-.02 \mathrm{~m}$ from the bottom of a graphite tube (Crucible, Saed/Manfredi G40, 1.5"OD x 1.25"ID x 3.75"DP) creating an approximately $.004-.005 \mathrm{~m}$ line on the side of the tube closest to the $\mathrm{S}$ pole of a gap magnet (any magnet providing required field strength would be sufficient), was incorporated with the basic apparatus (Tahan 2014) for D-brane trials as shown in Figure 1b. The laser was used to examine the possibility of the bending of spacetime resulting from gravitons emerging due to symmetry breaking (Tahan 2012) and interacting with the tube, imparting mass-energy thereby causing the tube to bend spacetime potentially sufficiently to alter the direction of the light. Sulfuric acid $\left(\mathrm{H}_{2} \mathrm{SO}_{4}\right)$ (typically $20 . \mathrm{mL}, 96 \%$ concd. Mallinckrodt Analytical Reagent, ACS) was in the tube for each experiment. Approximately $99.7 \mathrm{kPa}$ and $98-100 \%$ were the barometric pressure and relative humidity (Boston, MA, CW1378) during the largest laser light D-brane trial (Tahan 2011). The tube was 
clamped in the area of the static magnetic field measuring about 2000Gs, for consistency always in the direction of the $\mathrm{S}$ pole; $\mathrm{N}$ and $\mathrm{S}$ are the poles of the gap magnet. Wires from a breadboard to which the function generator was attached to a LED supplied $\approx 2 \mathrm{~Hz}(2.000-2.012 \mathrm{~Hz})$ and $\mathrm{V}_{\mathrm{p}-\mathrm{p}}=4.312-4.437$, predominately 4.375 , to the graphite vessel. The low frequency was not intended to alter Hydrogen alignment in the magnetic field. The set-up was ineffective when the magnet was removed. One wire with an alligator clip is required; three wires were connected triangularly to the top of the tube to ensure that the frequency and amplitude were being supplied--in view of potential inoperability as because of clip corrosion from acid exposure. The graphite tube, clamp that held the tube, and the stand to which the clamp was attached that included the $\approx .153 \mathrm{~kg}$ clamp holder were $\approx .098-.099 \mathrm{~kg}$, near $.230 \mathrm{~kg}$, and about $1.458 \mathrm{~kg}$ respectively. The set-up requires the frequency and amplitude, graphite tube, constant magnetic field strength $(\approx 2000 \mathrm{Gs})$, and concentrated sulfuric acid--the only components always needed. Different manners for supplying the parts can be tried, for example Hydrogen from a source other than $\mathrm{H}_{2} \mathrm{SO}_{4}$. But modifications to the components may be needed depending on the change: e.g. the amplitude may require adjustment. The graphite tube and acid supply should be changed periodically to avoid variables, for instance impurities in the acid or eventual deformation of the tube due to the acid that may prevent regular frequency conduction. The acid should be replaced in the tube for each trial unless an examiner wishes to test a particular aspect of the set-up involving the acid.

\section{Relative Humidity}

Watching the largest D-brane with an open string in the lab began the notion that the laser light was in a medium being dragged by the gradually increasingly rotating high gravitational area or black hole in the graphite tube of the symmetry breaking instrument (Tahan 2011). By that, the consequent deforming or twisting of the D-brane before the high gravitational area completely evaporated thereby resulting in the D-brane with the open string no longer being visible suggested the existence of a malleable medium, particularly when also seeing the bending of the laser light around the tube. The graphite tube gained mass-energy from gravitons that emerged due to the symmetry breaking process, which allowed for access to a fifth dimension of superparticles and gravitons (Tahan 2012) resulting in the sufficient bending of the suspected medium by the tube to alter the path of the light (Tahan 2011).

A primary curiosity from experimental conditions allowing for improved D-brane observations was the need for high relative humidity. Previous trials that may be discussed in a future manuscript led to the conclusion that a pushing influence accompanied the graviton pulling affect. Accordingly, the underlying structure of the light being the D-brane with the open string was thought to have appeared due to a particle, influential additionally in extra dimensions, that created what seemed as a push on objects. Specifically, the visualization of the D-brane and open string was due to the particle imparting influence particularly on the graphite tube thereby resulting in a pushing on the light, which is not different from the D-brane with the open string that is the light but simply typically is not seen being in the fifth dimension. Only the laser as collimated light is observable in the Standard Model visible sector. The push can be imagined to have created a figurative window to the fifth dimension, i.e. the influence pushed extra-dimensional spacetime. In other words, the resulting pushed light--essentially being held in place--exposed its fundamental structure D-brane and open string since the particle had influence on the extra dimension. So, how light is in the fifth dimension was seen. After the affect no longer existed as due to the black hole in the tube fully evaporating (Tahan 2011), spacetime returned to normal thereby eliminating visualization of the light in the fifth dimension. Uncertainty related to what was being pushed to allow for the visualization of the D-brane with the open string led to the initial thought that the air surrounding the tube had characteristics that would allow it to be influenced to show the D-brane.

The best observations having been with low barometric pressure and high relative humidity suggested that alternate scenarios should have allowed for similar visualizations of the D-brane, which did not occur. Since relative humidity is understood reduces the amount of light reaching a point (Knestrick, Cosden \& Curcio 1962)--relative humidity and light transmittance having been found inversely related (Ramirez-San-Juan, Choi, Franco, Nelson \& Aguilar 2005)--intuitively for the largest D-brane observations trials with low relative humidity should have allowed for the best D-brane visualizations since more light would have reached the point of influence, equating more light to a greater D-brane appearance. Trials supported that when less pressure was against the tube, influences from the tube would greater affect surroundings. Yet when barometric pressure and relative humidity were low, no complete D-branes with open strings for the laser light were observed.

The pushing influence from the tube was concluded to be a force with a different affect than gravitons, determined as with the mentioned previous trials--for instance experiments involving polarizers--suggesting that 
an additional particle that had photon characteristics accompanied the pulling force. The relative humidity had to be near a specific level for visualizations, in conjunction with a low barometric pressure (Tahan 2011). Thus, for the influences from the tube to have been affecting the air the thought became that a sufficient amount of pressure had to exist to permit an observed pushing, i.e. an influence on surroundings opposite to when the tube was affected by gravitons. But even median readings, e.g. near 100.pKa and $70 \%$, were not as productive for seeing D-branes with open strings.

Barometric pressure apparently had to be as low as possible to allow for D-brane observations, particularly when relative humidity was further from $100 \%$. In other words, the barometric pressure had to be uncharacteristically low $(\leq 99.7 \mathrm{kPa})$ for the best observations (Tahan 2011) since again high pressure seemingly acted as a force against the tube thereby countering particularly the pushing influence. And not even poorly visible D-branes were detected when relative humidity was too low (below 65-67\%), including when barometric pressure was low (Tahan 2011). Affects may still emerge with high barometric pressure and low relative humidity, but the conditions should not allow for effective influence of surroundings to produce easily visible D-branes. An apparatus to adjust barometric pressure and relative humidity was unavailable; the weather was relied upon. Therefore, an instrument allowing for the tuning of environmental settings would permit faster opportunities for trials. Additionally, though relative humidity is being discussed the region between the light source and the tube can include a different factor, synthetic or natural, that could affect photons similarly to allow for influences from the tube to be effective.

The understanding of high barometric pressure restricting influence from the tube brought to mind properties of the light, e.g. radiative pressure, that could have transferred to the air thereby contributing to the restricting of influences from the graphite tube. As the laser light traveled to the tube, radiative pressure was transferred to the air as photons were absorbed or scattered. Accordingly, higher relative humidity can be thought to have permitted greater transfer of photon properties to the air, creating a pressure against the tube thus limiting the influences from the tube on surroundings. The initial thought was that as the distance increased toward the tube, less photons from the light would exist so that less pressure would be in the air of high relative humidity at the point of influence from the graphite tube. The relative humidity along the path of the light from the consistent laser was invariant. Remembering the short distance $\approx .34-.35 \mathrm{~m}$ from the laser to the tube, radiative pressure at the point of influence that can be stated where the D-brane was observed should not have been significantly different compared to other points along the distance if considering the locations independently. The lack of significant radiative pressure difference can be understood by using an equation as the Beer-Lambert law with varying distances, which could lead to the amount of radiative pressure transferred to the air over the distance to be comprehended additive--for simplicity light lost to the air being linearly related to radiative pressure gained.

Since D-branes were not as well detected during low relative humidity when less radiative pressure would have transferred to the air, influence from the tube simply affecting the air at the point of influence seemed unlikely. By that, if the laser light was just passing in the air D-branes should have been seen equally as well or perhaps more often during days of low relative humidity. The notion emerged therefore that if the light was in a separate medium, radiative pressure could be lost from it so that at the point of influence the medium would present less pressure against the tube. In other words, due to the high relative humidity though the air would not be affected the other medium could be manipulated by influences from the graphite tube. At the point of influence enough photons would have been lost to allow for visible influence on the medium, again loss of photons meaning lower pressure. Thus, the Figure 1 set-up can be thought a system for functionality: low barometric pressure thereby less pressure on the tube, distance between the laser and tube being sufficient to allow enough light to be lost involving the high relative humidity, adequate radiative pressure loss from an additional medium to allow influences from the tube to be effective at the point of influence, the laser light frequency not being too high that could create a larger radiative pressure against the tube or insufficient radiative pressure loss, a pushing influence from the graphite tube emerging and affecting surroundings, the separate medium with an extra dimension being affected by an influence from the tube so that the D-brane with an open string can be observed.

The distance of the laser to the graphite tube accordingly cannot be too great considering photon loss so that at the point of influence insufficient photons would exist to allow for visualization of a laser light D-brane. A sufficient amount of radiative pressure loss was required, but the laser light should not be thought to have been an enormous radiative pressure source. Yet, what was lost from spacetime due to the high relative humidity was enough for observations. The need for low barometric pressure and the usefulness of high relative humidity supported that the influences from the tube responsible for the D-brane observations had particular character, i.e. being due to specific carrier particles.

This manuscript does not counter work disfavoring the need for a classical aether and does not indicate that the 
mechanical aether with motion exists. Simply, an explanation was needed for how the underlying foundation D-brane with an open string for the laser light could have appeared due to influences from the tube in consideration of environmental conditions. Specifically, gravity as the curvature of spacetime in General Relativity is known to create redshift, which was observed with the laser light consequently entering the graphite tube and interacting with the high gravitational area (Tahan 2011). The gravitons that emerged due to the symmetry breaking process (Tahan 2012), evidenced by the changed path of light due to the string bodies interacting with the tube of the apparatus (Tahan 2011), and related superparticles (spin 1 graviphoton, spin 0 graviscalar) do not interact directly with photons (Weinberg 1965, Srivastava \& Ashok 2005). Also, additional bosons from the tube would not have affected the photons of the laser light, e.g. photons from the evaporating high gravitational area in the tube (d'Enterria \& da Silveira 2013). Therefore, if the influences from the graphite tube could not have overcome the pressure in the air to push it the laser light was affected due to a push or relaxation from the tube on a separate medium in which it was traveling. When considering the D-brane and open string to be in an extra dimension (Tahan 2012, Tahan 2013), spacetime being a specific medium with an extra dimension is plausible. Consequently, if considering spacetime to be everywhere indicating that light is apart from the general relativity aether is difficult to imagine.

Scattering or absorption of the photons from the laser light also should be understood to have occurred relative to the barometric pressure, though for simplicity this manuscript simply discussed the high barometric pressure as a pressure or force of the air against the tube. Just understanding the pressure against the graphite tube to be greater when the barometric measure was higher was sufficient to communicate how influences from the tube interacted with surroundings. More importantly the notion of the barometric pressure needing to be low indicated that air and spacetime are independent mediums, though air is not separated from the ubiquitous spacetime. The high relative humidity allowed for radiative pressure loss to the air that was insufficient to prevent influences from the tube affecting spacetime. Specifically, consideration of the relative humidity allowed for air and spacetime to be visualized as mediums with distinct characteristics.

\section{Imparting Influence}

Gravitons and superparticles, specially graviphotons, have been mentioned encountered the graphite tube thereby affecting spacetime. Thus, the notion of imparting influence should be remembered is not restricted to photons but can be considered for any string body. If focusing on the photon and considering a rate of photons (photons/s) to have encountered an area (meters $(\mathrm{m})$ ) over a specific time the interaction would result in the appearance of force: (photons/s)m/s. Any string can be inserted--e.g. gravitons/s--to illustrate the force imparted from the body. In other words, when recalling that the string is synonymous to energy (Tahan 2011) visualizing the imparting of energy as a string is in contact with a medium allows the equivalence to be imagined better when incorporating the velocity of the string. The string becomes understood to have measurable movement in consideration of its velocity in spacetime $\left(\mathrm{E} / \mathrm{c}^{2} \rightarrow\right.$ the string body). Consequently, (photons $) \mathrm{m} / \mathrm{s}^{2}$ illustrates that energy and force exist due to the string, apart from permitting the comprehension that strings can be used to transfer physical properties.

The mention of the string being responsible for force should not be considered to disregard the individual properties of strings, particularly regarding resultant particles. The understanding of the string as energy with velocity allows for better appreciation of the body interacting with surfaces including other strings, e.g. branes, so that the orientation or how the string body encounters an object is appreciated important to imparted character. Strings could be affected differently by other strings depending on orientation related to branes. For instance, gravitons may be more influential on a D0-brane than on open strings ending on different branes or open strings ending on the same brane, as due to the distance from gravitons and superparticles. Accordingly, particles may be observed to be affected uniquely primarily due to how the strings for the particles exist related to branes and the influencing gravitons in the fifth dimension. Confounding cosmological observations involving particles could be explained simply as the bodies having been affected differently due to how the strings for the particles exist, e.g. photons being open strings on D-branes, especially related to surroundings. Also, the open string of the D-brane in Figure 1 seemed not to have both ends on branes. The observation permitted the image of spacetime being apart from a brane though related to it, as with holography and a two dimensional surface ('t Hooft 1993, Bekenstein 1994, Susskind 1995, Tahan 2013), so that strings may connect to or end on spacetime.

The ability to manipulate spacetime with influences from the tube thus backed the medium to have material structure and properties, principally mass-energy. Trials supported that branes have tension, which suggested the bodies to be limited areas. Therefore, imparted energy would be restricted in terms of how much energy can be transferred to particular points; a distance can only accept a definite amount of energy before the brane distorts or bends. Observations in trials--e.g. the curving of the laser light around the tube, the frame dragging involving 
the D-brane (Tahan 2011), pushing and pulling observations between objects--supported spacetime to have a distinct, consistent tension that is not too great to prevent spacetime bending by imparting gravitons to objects. Thus, all bodies can be imagined to be suspended in spacetime and to curve it depending on individual mass-energy.

What should be remembered is that the size of the laser light D-brane with the open string was relative to the amount of energy imparted to it (Tahan 2013). In other words, the size depended on the energy--gravitons and superparticles--interacting with the D-brane, just as the size of any D-brane can change due to imparted energy. The largest D-brane from trials as seen in Figure 1 was recorded since more energy had been imparted from the high gravitational area to the graphite tube that influenced surrounding spacetime, transferring energy to spacetime and thereby also to the D-brane due to being in the environment. By that, the laser light D-brane with an open string had to have been in a medium to which the energy imparted to the graphite tube could have transferred to interact with it. Accordingly, accessing the fifth dimension of gravitons and superparticles through string experimentation (Tahan 2014) involving the Hydrogen brane was supportive of AdS/CFT correspondence.

\section{Conclusions}

Apart from restating observations from trials with the Figure 1 instrument, e.g. the deforming of the D-brane with the open string due to a rotating high gravitational area in the graphite tube (Tahan 2011), simple consideration of environmental conditions particularly high relative humidity when the largest D-brane for the laser light was observed requires acceptance of spacetime to be a specific, malleable medium in which light travels. Again expression of spacetime as a general relativity aether is unoriginal, though this work is the first to make the conclusion based on laboratory experimentation. Acceptance of spacetime as a tangible environment would be helpful for physics discussions, for example providing a reference frame in explanations for quantum phenomena (Davies \& Brown 1993), and should encourage thoughts of practical applications, including involving bending the medium with use of the Figure 1 apparatus while remembering conclusions for a brane--particularly about tension that can be altered (Tahan 2014). In other words, spacetime and string theory including resultant quantum events should not be thought separate.

\section{References}

Bekenstein, J. D. (1994). Entropy bounds and black hole remnants. Phys. Rev., 49D, 1912-1921. http://dx.doi.org/10.1103/PhysRevD.49.1912

David, d'E., \& da Silveira, G. G. (2013). Observing light-by-light scattering at the Large Hadron Collider. Phys. Rev. Lett., 111, 080405. http://dx.doi.org/10.1103/PhysRevLett.111.080405

Davies, P. C. W., \& Brown, J. R. (1993). The ghost in the atom: A discussion of the mysteries of quantum physics (John Bell), Cambridge: Cambridge University Press.

Einstein, A. (1905). Ist die Trägheit eines Körpers von seinem Energieinhalt abhängig? (Does the inertia of a body depend upon its energy-content?). Annalen der Physik, 18, 639-643. http://dx.doi.org/10.1002\%2Fandp.19053231314

Einstein, A. (1920). Äther und Relativitätstheorie (Ether and the theory of relativity), An Address delivered on May 5th, 1920, in the University of Leyden, Berlin: Springer.

Einstein, A. (1924). Über den Äther (Concerning the aether). Verhandlungen der Schweizerischen Naturforschenden Gesellschaft, 105(2), 85-93.

Hooft, G. T. (1993). Dimensional reduction in quantum gravity. arXiv: gr-qc/9310026.

Kaluza, T. (1921). Zum Unitätsproblem in der Physik. Sitzungsber. Preuss. Akad. Wiss. Berlin. (Math. Phys.), 966-972. http://archive.org/details/sitzungsberichte1921 preussi

Klein, O. (1926). Quantentheorie und fünfdimensionale Relativitätstheorie (Quantum theory and five dimensional theory of relativity). Zeitschrift für Physik A, 37(12), 895-906. http://dx.doi.org/10.1007/BF01397481

Knestrick, G. L., Cosden, T. H., \& Curcio, J. A. (1962). Atmospheric scattering coefficients in the visible and infrared regions. JOSA, 52(9), 1010-1016. http://dx.doi.org/10.1364/JOSA.52.001010

Laughlin, R. B. (2005). A Different Universe: Reinventing Physics from the Bottom Down. New York: Basic Books.

Michelson, A. A., \& Morley, E.W. (1887). On the relative motion of the Earth and the luminiferous ether. American Journal of Science, 34, 333-345. http://dx.doi.org/10.2475/ajs.s3-34.203.333 
Minkowski, H. (1908). Die Grundgleichungen für die elektromagnetischen vorgänge in bewegten körpern (The fundamental equations for electromagnetic processes in moving bodies), Nachrichten von der Gesellschaft der Wissenschaften zu Göttingen, Mathematisch-Physikalische Klasse, 53-111.

Minkowski, H. (1909). Raum und zeit (Space and time). Physikalische Zeitschrift, 10, 75-88.

Ramirez-San-Juan, J. C., Choi, B., Franco, W., Nelson, J. S., \& Aguilar, G. (2005). Effects of relative humidity on laser light transmittance during cryogen spray cooling of in vitro skin phantoms, Proc. SPIE 5686. Photonic Therapeutics and Diagnostics, 154. http://dx.doi.org/10.1117/12.591197

Shankland, R. S. (1964). Michelson-Morley experiment. American Journal of Physics, 31(1), 16-35. http://dx.doi.org/10.1119/1.1970063

Srivastava, R. K., \& Ashok, J. (2005). Statistical Mechanic. New Delhi: PHI Learning Pvt. Ltd.

Susskind, L. (1995). The world as a hologram. J. Math. Phys., 36, 6377-6396. http://dx.doi.org/10.1063/1.531249

Tahan, A. C. (2011). Exposing strings in the laboratory with a novel technique. Appl. Phys. Res., 3(2), 39-51, http://dx.doi.org/10.5539/apr.v3n2p39

Tahan, A. C. (2012). Diagrammatic presentation for the production of gravitons and supersymmetry. Mod. Appl. Sci., 6(9), 76-83. http://dx.doi.org/10.5539/mas.v6n9p76

Tahan, A. C. (2013). Low mass gravitino: Re-introducing the superpartner as dark matter with consideration to inflation due to experimentation. Mod. Appl. Sci., 7(12), 43-55. http://dx.doi.org/10.5539/mas.v7n12p43

Tahan, A. C. (2014). BICEP2 and the gravitino mass: The questionable result. Mod. Appl. Sci., 8(5), 30-35. http://dx.doi.org/10.5539/mas.v8n5p30

Weinberg, S. (1965). Photons and gravitons in perturbation theory: Derivation of Maxwell's and Einstein's equations. Phys. Rev., 138, B988-B1002. http://dx.doi.org/10.1103/PhysRev.138.B988

\section{Copyrights}

Copyright for this article is retained by the author(s), with first publication rights granted to the journal.

This is an open-access article distributed under the terms and conditions of the Creative Commons Attribution license (http://creativecommons.org/licenses/by/3.0/). 